Originalien

Unfallchirurg 2020 $123: 711-723$

https://doi.org/10.1007/s00113-020-00777-8

Online publiziert: 5. März 2020

(c) Der/die Autor(en) 2020

\section{Redaktion}

W. Mutschler, München

H. Polzer, München

B. Ockert, München

J. Schimrigk' $\cdot$ C. Baulig ${ }^{2} \cdot$ C. Buschmann ${ }^{3,4} \cdot$ J. Ehlers ${ }^{1}$ C. Kleber ${ }^{4,5} \cdot$ S. Knippschild ${ }^{2}$. B. A. Leidel ${ }^{6} \cdot$ T. Malysch $^{7} \cdot$ E. Steinhausen ${ }^{8,9} \cdot$ J. Dahmen $^{8,9,10}$

'Lehrstuhl für Didaktik und Bildungsforschung im Gesundheitswesen, Department Humanmedizin, Fakultät für Gesundheit, Universität Witten/Herdecke, Witten/Herdecke, Deutschland; ${ }^{2}$ Institut für Medizinische Biometrie und Epidemiologie (IMBE), Department Humanmedizin, Fakultät für Gesundheit, Universität Witten/Herdecke, Witten/Herdecke, Deutschland; ${ }^{3}$ Institut für Rechtsmedizin, Charité Universitätsmedizin Berlin, Corporate Member of Freie Universität Berlin, Humboldt-Universität zu Berlin and Berlin Institute of Health, Berlin, Deutschland; ${ }^{4}$ AG Trauma, Deutscher Rat für WiederbelebungGerman Resuscitation Council (GRC), Ulm, Deutschland; ${ }^{5}$ Chirurgische Notaufnahme,

Universitätszentrum für Orthopädie \& Unfallchirurgie, Universitätsklinikum TU Dresden, Dresden, Deutschland; ' ${ }^{6}$ Zentrale Notaufnahme, Campus Benjamin Franklin, Charité - Universitätsmedizin Berlin, Berlin, Deutschland; ' Klinik für Anästhesiologie und Intensivtherapie, Klinikum Brandenburg, Medizinische Hochschule Brandenburg, Brandenburg, Deutschland; ${ }^{8} \mathrm{Klinik}$ für Orthopädie und Unfallchirurgie, BG Klinikum Duisburg, Duisburg, Deutschland; ${ }^{9}$ Ärztliche Leitung Rettungsdienst Berlin, Fakultät für Gesundheit, Department Humanmedizin, Universität Witten/Herdecke, Witten, Deutschland; ${ }^{10}$ Ärztliche Leitung Rettungsdienst, Berliner Feuerwehr, Berlin, Deutschland

\title{
Indikation, Prozedere und Outcome der präklinischen Notfallthorakotomie - eine systematische Literaturrecherche
}

der Registerforschung, der klinischen Forschung sowie Erkenntnisse aus dem Bereich der Unfallprävention. Aufgrund von wenigen verfügbaren, uneinheitlichen und unvollständigen Daten gab es lange Zeit keine oder wenige wissenschaftliche Erkenntnisse und darauffolgende Neuerungen im Bereich der präklinischen Traumaversorgung. Insbesondere Patienten, die bereits präklinisch verstarben, wurden meist nicht näher in der wissenschaftlichen Betrachtung erfasst bzw. in die Weiterentwicklung neuer, insbesondere präklinischer Behandlungsstrategien eingeschlossen.

Neben der Verbesserung der präklinischen Versorgungsqualität durch systematische und fundierte Schulungen, kontinuierliche Qualitätssicherung und insbesondere die Vermeidung überproportional häufiger Behandlungsfehler [4] stehen neuere Behandlungsstrategien insbesondere im Bereich der Reanimation nach Trauma im Vordergrund. Galten Traumareanimationen lange Zeit als weitestgehend aussichtslos und mit schlechter Prognose vergesellschaftet, konnten Zwingmann et al. inzwischen

\footnotetext{
Kernaussagen

- Die Überlebensrate bei (stumpfem oder penetrierendem) traumatischem Herz-Kreislauf-Stillstand nach durchgeführter PHRT beträgt für alle 205 eingeschlossenen PHRT-Patienten $12 \%$. Eine Differenzierung zwischen dem Outcome der verschiedenen Verletzungsmechanismen konnte innerhalb dieses Reviews nicht untersucht werden. Ein Überlebensvorteil der penetrierenden Verletzungen ist jedoch anzunehmen.

- $75 \%$ der PHRT-Patienten, die aus dem Krankenhaus entlassen wurden, haben ein gutes neurologisches Outcome.

- Eine PHRT kann auch von „nichtchirurgischen" Notärzten durchgeführt werden.

- Neben "skills trainings" sind insbesondere Team-Trainings und an regionalen Strukturen orientierte SOP geeignet, eine gute PHRT-Versorgung zu implementieren.

- Da viele der eingeschlossenen Publikationen niedrige Fallzahlen beinhalten, ist das Verzerrungspotenzial der einzelnen Publikationen sowie auch dieses Reviews hoch. Weitere systematische Forschung im Bereich der präklinischen Traumareanimation ist insbesondere auch zur Umsetzung der ERC-Leitlinien-Vorgaben zwingend erforderlich.
} 


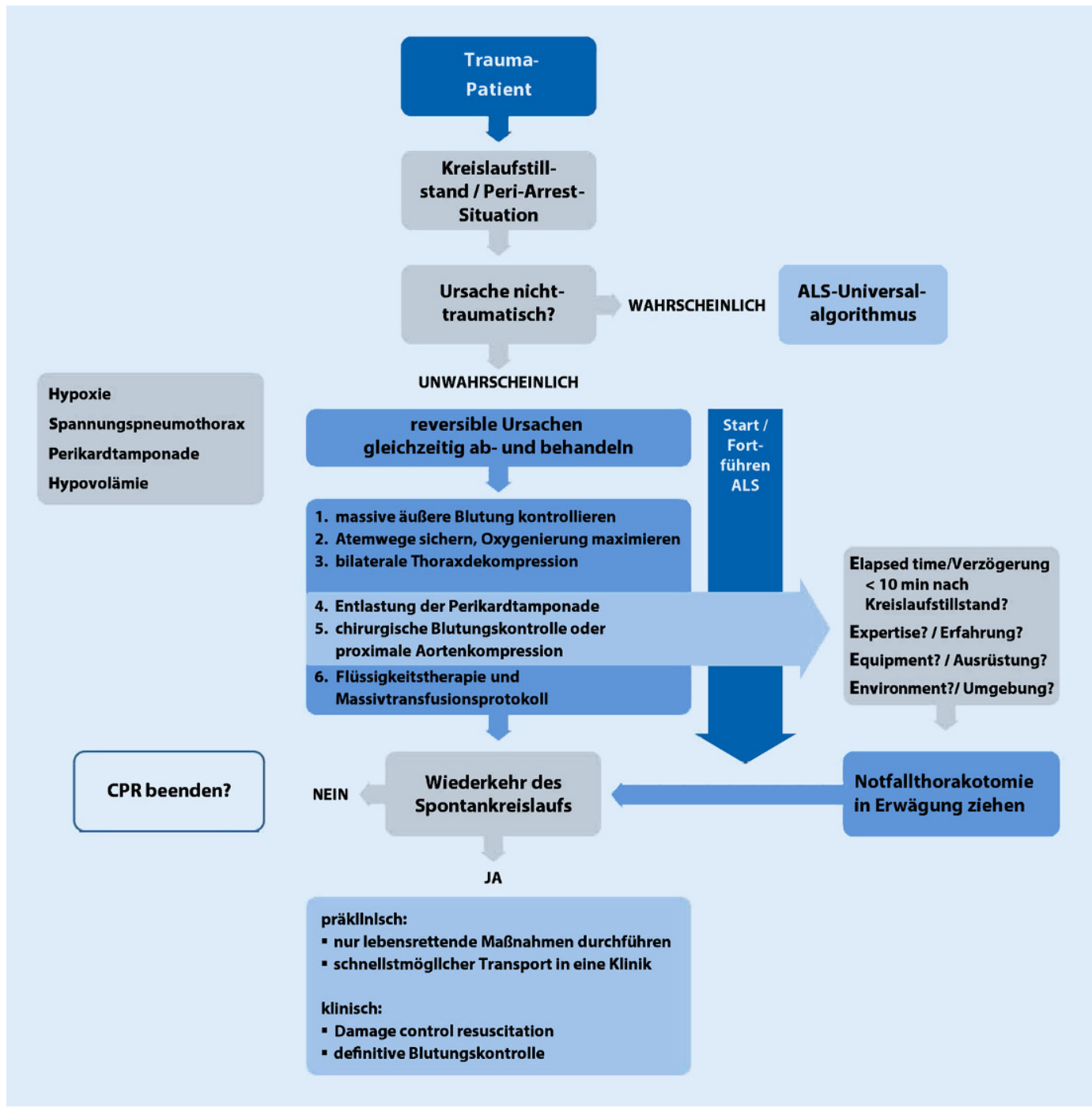

Abb. $1<$ Algorithmus traumatischer Herz-Kreislauf-Stillstände des European Resuscitation Council [6]. (Abb. aus [7] unter Berücksichtigung von [8] (c) German Resuscitation Council (GRC) und Austrian Resuscitation Council (ARC) 2015. ALS „Advanced Life Support", CPR "Cardiopulmonary Resuscitation" für Deutschland zeigen, dass bis zu $32 \%$ aller Patienten, die nach schwerer Verletzung präklinisch reanimationspflichtig werden, dies überlebten, und dass $15 \%$ dieser Überlebenden das Krankenhaus mit einem guten neurologischen Ergebnis verließen [5].

Vor dem Hintergrund dieser und ähnlicher neuerer wissenschaftlicher Erkenntnisse beinhalten seit 2015 die Reanimationsleitlinien des European Resuscitation Council (ERC) einen eigenständigen Abschnitt und Algorithmus für den traumatischen Herz-KreislaufStillstand (• Abb. 1; [6]). Wesentliche Änderungen im Vergleich zu vorangegangenen Versionen der Reanimationsleitlinien sind im Kontrast zu dem Standard-ACLS-Algorithmus die Empfehlung, dass bei der Traumareanimation prioritär alle reversiblen Ursachen sofort behoben werden sollen, und dass die Durchführung der klassischen Thoraxkompression weitestgehend ineffektiv bleibt (und deshalb sogar nachrangig ist), wenn die reversiblen Ursachen (- Infobox 1) nicht umgehend behandelt werden („you can't pump an empty or obstructed heart").

Während für die äußere Blutungskontrolle, die Beatmung, Thoraxdekompression und Volumensubstitution etablierte Verfahrensanweisungen und entsprechende Schulungsprogramme für das ärztliche und nichtärztliche Personal im Rettungsdienst zur Verfügung stehen [4, $9,10]$, ist die präklinische Thorakotomie („prehospital resuscitative thoracotomy“ PHRT) als notfallmedizinisches Therapieverfahren im deutschsprachigen Raum weder etabliert noch systematisch geschult. Seit 2015 soll gemäß ERC-
Leitlinie eine Notfallthorakotomie in Erwägung gezogen werden (• Abb. 1), wenn bestimmte Indikationskriterien und Voraussetzungen erfüllt sind [6]. Ziel der PHRT ist gemäß ERC-Leitlinie die Therapie reversibler Ursachen des Kreislaufstillstands wie z.B. thorakaler Blutungen, Perikardtamponade oder zur proximalen Aortenkompression [7, 11, 12].

Vor dem Hintergrund der großen Differenz zwischen Leitlinienempfehlungen und der im deutschsprachigen Raum angewandten notfallmedizinischen Behandlungspraxis untersucht das vorliegende Review erstmals systematisch sämtliche publizierten Daten zum Outcome von Patienten nach PHRT. Ziel des Reviews ist es ferner, einen Überblick über die bis dato publizierten Indika- 
Unfallchirurg 2020 $\cdot 123: 711-723$ https://doi.org/10.1007/s00113-020-00777-8

(c) Der/die Autor(en) 2020

\section{J. Schimrigk · C. Baulig · C. Buschmann · J. Ehlers · C. Kleber · S. Knippschild · B. A. Leidel · T. Malysch · E. Steinhausen · J. Dahmen Indikation, Prozedere und Outcome der präklinischen Notfallthorakotomie - eine systematische
Literaturrecherche}

\section{Zusammenfassung}

Hintergrund. Die präklinische Notfallthorakotomie („prehospital resuscitative thoracotomy", PHRT) ist eine von den aktuellen Reanimationsleitlinien des European Resuscitation Council (ERC) empfohlene, kontrovers diskutierte Maßnahme zur Akutbehandlung des traumatischen Herz-Kreislauf-Stillstands („traumatic cardiac arrest“, TCA). Ziel dieser Arbeit ist das vollständige Abbilden und Zusammenfassen der vorhandenen Literatur mit der zugrunde liegenden Hypothese, dass vorhandene Publikationen die Machbarkeit und das Überleben nach PHRT bei Patienten mit TCA mit gutem neurologischen Outcome zeigen. Methode. Es erfolgte eine systematische Literaturrecherche in den Datenbanken PubMed, EMBASE, Google Scholar, SpringerLink und Cochrane. Die Studienauswahl, Datenextraktion und Bewertung des Verzerrungspotenzials wurden unabhängig von 2 Autoren durchgeführt. Als primärer Endpunkt wurde das Outcome der Patienten mit TCA nach präklinischer Notfallthorakotomie gewählt.

Ergebnisse. Es wurden 4616 Publikationen gesichtet, 21 Veröffentlichungen mit insge- samt 287 Patienten konnten eingeschlossen werden. Für eine detaillierte deskriptive Analyse eigneten sich 15 Publikationen mit insgesamt 205 Patienten. Der TCA dieser Patienten war am häufigsten durch eine Perikardtamponade, thorakale Gefäßverletzungen und schwere extrathorakale Mehrfachverletzungen verursacht. In $24 \%$ der Fälle trat ein TCA im Beisein des Notarztes auf. Die "Clamshell“-Thorakotomie (53\%) wurde präklinisch häufiger als die anterolaterale Thorakotomie (47\%) angewandt. $12 \%$ (25/205) der PHRT-Patienten nach TCA verließen lebend das Krankenhaus, 9\% $(n=19 / 205)$ mit gutem und $1 \%(n=3 / 205)$ mit schlechtem neurologischen Outcome (nach Glasgow Outcome Scale, GOS). Schlussfolgerung. Die Prognose des TCA scheint deutlich besser zu sein als lange angenommen. Maßgeblich für den Erfolg von Reanimationsbemühungen beim TCA scheint die unverzügliche, teils invasive Therapie aller reversiblen Ursachen zu sein. Die von der ERCReanimationsleitlinien für den TCA empfohlenen Maßnahmen scheinen insbesondere für die Präklinik bisher unzureichend umgesetzt.
Eine besondere Kontroverse hinsichtlich der Empfehlungen der Leitlinie besteht zur Frage, ob eine PHRT erfolgreich angewandt werden kann und deren flächendeckende Einführung in Deutschland sinnvoll erscheint. Die vorliegende systematische Übersichtsarbeit unterstreicht trotz Leitlinienempfehlung den Mangel an hochwertiger Evidenz zur PHRT, wobei eine Überlebenswahrscheinlichkeit bis zur Entlassung aus dem Krankenhaus von $12 \%$, in $75 \%$ davon mit gutem neurologischen Outcome berichtet wird. Das Verzerrungspotenzial der Ergebnisse der einzelnen Publikationen sowie auch dieses Reviews ist hoch. Weitere systematische Forschung im Bereich der präklinischen Traumareanimation ist insbesondere auch zur Akzeptanz der Leitlinienvorgaben erforderlich.

\section{Schlüsselwörter}

„Prehospital resuscitative thoracotomy“ . „Preclinical emergency resuscitative thoracotomy" · Thorakotomie · Präklinisch . Perikardtamponade · Traumatischer Herz-Kreislauf-Stillstand - Thoraxtrauma . Notfallmedizin

\section{Indications, procedure and outcome of prehospital emergency resuscitative thoracotomy-a systematic literature search}

\section{Abstract}

Background. Prehospital resuscitative thoracotomy (PHRT) is a controversially discussed measure for the acute treatment of traumatic cardiac arrest (TCA) recommended by the current guidelines of the European Resuscitation Council (ERC). The aim of this work is the comprehensive presentation and summary of the available literature with the underlying hypothesis that the available publications show the feasibility and survival following PHRT in patients with TCA with a good neurological outcome.

Method. A systematic literature search was performed in the databases PubMed, EMBASE, Google Scholar, Springer LINK and Cochrane. The study selection, data extraction and evaluation of bias potential were performed independently by two authors. The outcome of patients with TCA after PHRT was selected as the primary endpoint.

Results. A total of 4616 publications were found of which 21 publications with a total of 287 patients could be included in the analyses. For a detailed descriptive analysis,
15 publications with a total of 205 patients were suitable. The TCA of these patients was most commonly caused by pericardial tamponade, thoracic vascular injuries and severe extrathoracic multiple injuries. In $24 \%$ of the cases TCA occurred in the presence of the emergency physician. Clamshell thoracotomy (53\%) was used preclinically more often than anterolateral thoracotomy (47\%). Of the PHRT patients after TCA 12\% $(25 / 205)$ left the hospital alive, $9 \%(n=19 / 205)$ with good neurological outcome and $1 \%$ $(n=3 / 205)$ with poor neurological outcome (according to the Glasgow outcome scale, GOS).

Conclusion. The prognosis of TCA seems to be much better than has long been assumed. Decisive for the success of resuscitation efforts in TCA seems to be the immediate, partly invasive treatment of all reversible causes. The measures for TCA recommended by the ERC resuscitation guidelines, seem to be poorly implemented, especially in the preclinical setting. A controversy regarding the recommendations of the guidelines is the question of whether a PHRT can be successfully implemented and if the comprehensive introduction in Germany seems to be meaningful. Despite the recommendation of the guidelines, this systematic review and meta-analysis underlines the lack of highquality evidence on PHRT, whereby a survival probability to hospital discharge of $12 \%$ was reported, of which $75 \%$ had a good neurological outcome. The risk of bias of the results in individual publications as well as in this review is high. Further systematic research in the field of preclinical trauma resuscitation is particularly necessary also for acceptance of the guidelines.

\section{Keywords}

Prehospital resuscitative thoracotomy . Preclinical emergency resuscitative thoracotomy - Thoracotomy · Preclinical · Pericardial tamponade - Traumatic cardiac arrest . Thoracic injuries - Emergency medicine 


\section{Infobox 1 Zur präklinischen Notfallthorakotomie}

\section{Indikation}

Die ERC-Reanimationsleitlinie 2015 fordert die sofortige Therapie aller reversiblen Ursachen des "traumatic cardiac arrest" (TCA, [6]):

Reversible Ursachen des TCA sind:

1. Hypoxie

2. Spannungspneumothorax

3. Hypovolämie

4. Perikardtamponade

Maßnahmen beim TCA

c Stoppen kritischer Blutungen durch Kompressions-/Tamponierungsverbände, Tourniquets und Beckenschlinge

A. Oxygenierung/Intubation zu Therapie/Ausschluss traumainduzierter Apnoen

B. Fingerthorakostomie zu Therapie/Ausschluss eines Spannungspneumothorax

C. Volumensubstitution (vorzugsweise Blut) zu Therapie/Ausschluss Hypovolämie bzw. eines Vorlastmangels via intravenöse oder intraossäre Zugänge oberhalb des Bauchnabels (bei stumpfen Traumata von Bauch/Becken bzw. ggf. beim penetrierenden Trauma auch über andere zentrale Zugänge z. B. in der Leiste)

"Clamshell thoracotomy" (PHRT) zur Therapie der Perikardtamponade sowie schweren intraund extrathorakalen Blutungen (direkte Blutungskontrolle, Aortenkompression zur indirekten Blutungskontrolle und zur Erhöhung des peripheren Widerstands und Verbesserung der Perfusion von Herz/Hirn sowie direkte Herzmassage)

\section{Voraussetzungen}

Im Fall eines TCA soll bei erfolgloser Therapie aller anderen reversiblen Ursachen die Durchführung einer präklinischen Notfallthorakotomie („prehospital resuscitative thoracotomy“, PHRT) in Erwägung gezogen werden, wenn seit dem Vorliegen der letzten Vitalzeichen (z. B. jeglicher elektrischer Aktivität im Elektrokardiographie, jeder Form der Atemaktivität, Reaktion auf Schmerzreiz) weniger als 10 min bei stumpfem Verletzungsmuster bzw. weniger als 15 min bei penetrierenden, thorakalen Verletzungen vergangen sind. Weitere Voraussetzungen sind gemäß ERC-Leitlinie das Vorhandensein des notwendigen Equipments, die notwenige Expertise (Ausbildung) und eine sichere Umgebung (Environment) [6].

\section{Durchführung der PHRT}

Die PHRT hat die unverzügliche Eröffnung des Thorax am Unfallort zum Ziel, um das Perikard zu entlasten, Blutungen zu stillen und mittels proximaler Aortenkompression einen vorübergehenden Kreislauf herzustellen. Geschultes ärztliches Personal jeder Fachrichtung kann diese Maßnahme durchführen [13]. Mit einfachen Notfallthorakotomie-Sets für den Rettungsdienst (Skalpell, Pinzette, stabile Trauma- und Präparierschere, Klemmen und ggf. Gigli-Säge) lässt sich der Thorax so uni- oder bilateral öffnen (Clamshell- oder anterololaterale Thorakotomie). Ausgehend von den bilateralen Thorakostomiestellen im 4./5. Interkostalraum in der Medioaxillarlinie zur Thoraxdekompression im Rahmen der Reanimation kann die Schnittführung zur PHRT im Rippenzwischenraum bis zum Sternum erweitert werden. Das Sternum kann im Fall der Clamshell-Thorakotomie mittels stabiler Trauma-Schere oder Gigli-Säge schnell und einfach durchtrennt werden und so der Zugang zu den Thoraxorganen und der Therapie der potenziell reversiblen Ursachen des TCA ermöglicht werden.

tionen, Behandlungsstrategien und die verfügbare Evidenz zu geben.

\section{Methode}

Das vorliegende systematische Review wurde nach den Empfehlungen der Cochrane Collaboration für systematische Übersichtsarbeiten erstellt [14]. Die Systematik der Publikation orientierte sich an der PRISMA-Checkliste, um eine umfassende und verständliche Berichterstattung zu ermöglichen [15].

\section{Literatursuche}

Es wurde eine systematische Recherche nach definierten Ein- und Ausschlusskriterien in 5 internetbasierten Datenbanken (PubMed, EMBASE, Google Scholar, SpringerLink und Cochrane) durchgeführt. Der primäre Endpunkt wurde im Vorhinein mithilfe des PI$\mathrm{CO}$ (Patient/Intervention/Comparison/ Outcome)-Schemas als „Überleben des Patienten bis zur Krankenhausaufnahme" sowie als sekundärer Endpunkt das „neurologische Outcome der Pati- enten nach PHRT zum Zeitpunkt der Krankenhausentlassung" definiert.

Die Suchstrategie wurde aus definierten Suchbegriffen und deren Kombination mittels Bool-Operatoren durchgeführt. Diese, sowie die vollständige Suchhistorie sind als zusätzliches Onlinematerial dokumentiert und dort einsehbar. Die Literaturrecherche wurde bis zum 31.12.2018 durchgeführt).

\section{Ein- und Ausschlusskriterien}

Um eine möglichst große Sensitivität der Suche zu gewährleisten, wurde die Recherche weder durch eine Limitierung hinsichtlich des Datums der Veröffentlichung noch durch das Studiendesign eingeschränkt. Der Ein- bzw. Ausschluss der Publikationen erfolgte stufenweise durch Sichtung der Titel, Sichtung der Abstracts und Sichtung der Volltexte (einsehbar als zusätzliches Onlinematerial)

Eingeschlossen wurden sämtliche englisch- oder deutschsprachigen Publikationen, die Zahlen zur Maßnahme "präklinische Thorakotomie" lieferten und bis zum 31.12.2018 veröffentlicht wurden ( $\bullet$ Tab. 1). Publikationen, die nicht als „peer reviewed publications“ veröffentlicht wurden, fanden keine Berücksichtigung in dieser Untersuchung. Ebenso ausgeschlossen wurden Übersichtsartikel zur PHRT, welche im Rahmen der Literatursuche gefunden wurden. Die Primärquellen aller Übersichtsarbeiten wurden systematisch nach zusätzlicher PHRT-Literatur durchsucht. Hiernach wurden keine zusätzlichen Publikationen identifiziert.

\section{Datenextraktion und Bewertung}

Die Datenextraktion erfolgte durch 2 unabhängige Reviewer mittels Sichtung der Volltexte, wobei folgende Informationen in einem zuvor erstellten ExcelErhebungsbogen dokumentiert wurden: Anzahl der Patienten hinsichtlich Unfallmechanismus, Ursache des traumatischen Herz-Kreislauf-Stillstands, verschiedene Zeiträume innerhalb der Rettungskette, Art der Thorakotomie, jeweiliges Fachgebiet der durchführenden Ärzte sowie das Outcome. 
Tab. 1 Übersicht der eingeschlossenen Publikationen zur „prehospital resuscitative thoracotomy“

\begin{tabular}{|c|c|c|c|c|}
\hline Autor & Titel & Land & $\begin{array}{l}\text { Erscheinungs- } \\
\text { jahr }\end{array}$ & Fallzahl \\
\hline Davies et al. [16] & $\begin{array}{l}\text { Thirteen Survivors of Prehospital Thoracotomy for Penetrating Trauma: } \\
\text { A Prehospital Physician-Performed Resuscitation Procedure That Can Yield } \\
\text { Good Results }\end{array}$ & $\begin{array}{l}\text { England } \\
\text { (London) }\end{array}$ & 2011 & 71 \\
\hline Athanasiou et al. [17] & $\begin{array}{l}\text { Emergency thoracotomy in the pre-hospital setting: a procedure requiring } \\
\text { clarification }\end{array}$ & $\begin{array}{l}\text { England } \\
\text { (London) }\end{array}$ & 2004 & 31 \\
\hline Coats et al. [18] & $\begin{array}{l}\text { Prehospital Resuscitative Thoracotomy for Cardiac Arrest after Penetrating } \\
\text { Trauma: Rationale and Case Series }\end{array}$ & $\begin{array}{l}\text { England } \\
\text { (London) }\end{array}$ & 2001 & 39 \\
\hline Corral et al. [19] & A successful emergency thoracotomy performed in the field & Spanien & 2007 & 6 \\
\hline Craig et al. [20] & On scene thoracotomy: a case report & $\begin{array}{l}\text { England } \\
\text { (London) }\end{array}$ & 1999 & 1 \\
\hline Keogh et al. [21] & $\begin{array}{l}\text { Survival following pre-hospital arrest with on-scene thoracotomy for } \\
\text { a stabbed heart }\end{array}$ & $\begin{array}{l}\text { England } \\
\text { (London) }\end{array}$ & 1996 & 1 \\
\hline Matsumoto et al. [22] & $\begin{array}{l}\text { Role of resuscitative emergency field thoracotomy in the Japanese helicop- } \\
\text { ter emergency medical service system }\end{array}$ & Japan & 2009 & 34 \\
\hline McKenzie et al. [23] & $\begin{array}{l}\text { A Case of Prehospital Traumatic Arrest in a US Special Operations Soldier: } \\
\text { Care from Point of Injury to Full Recovery }\end{array}$ & Unbekannt & 2016 & 1 \\
\hline Puchwein et al. [24] & Erfolgreiche präklinische Thorakotomie bei einem 17-jährigen Mann & Österreich & 2013 & 1 \\
\hline Purkiss et al. [25] & $\begin{array}{l}\text { Efficacy of urgent thoracotomy for trauma in patients attended by a heli- } \\
\text { copter emergency medical service }\end{array}$ & $\begin{array}{l}\text { Schottland } \\
\text { (Edinburgh) }\end{array}$ & 1994 & 9 \\
\hline Rudolph et al. [26] & Clamshell-Thorakotomie nach thorakalen Messerstichen & Deutschland & 2017 & 1 \\
\hline $\begin{array}{l}\text { Van Vledder et al. } \\
\text { [27] }\end{array}$ & $\begin{array}{l}\text { Out of hospital thoracotomy for cardiac arrest after penetrating thoracic } \\
\text { trauma }\end{array}$ & Niederlande & 2017 & 33 \\
\hline Wall et al. [28] & $\begin{array}{l}\text { Successful roadside resuscitative thoracotomy: case report and literature } \\
\text { review }\end{array}$ & USA & 1994 & 1 \\
\hline Wright et al. [29] & Cardiac tamponade: a case of kitchen floor thoracotomy & $\begin{array}{l}\text { England } \\
\text { (London) }\end{array}$ & 2002 & 1 \\
\hline $\begin{array}{l}\text { Hachimi-Idrissi et al. } \\
\text { [30] }\end{array}$ & Open chest cardiopulmonary resuscitation in out-of-hospital cardiac arrest & Belgien & 1997 & 33 \\
\hline Dahmen et al. [31] & $\begin{array}{l}\text { Erfolgreiche präklinische Notfallthorakotomie nach stumpfem Thoraxtrau- } \\
\text { ma }\end{array}$ & Deutschland & 2018 & 1 \\
\hline Lockey et al. [32] & Traumatic Cardiac Arrest: Who Are the Survivors? & $\begin{array}{l}\text { England } \\
\text { (London) }\end{array}$ & 2006 & 9 \\
\hline Belezia et al. [33] & $\begin{array}{l}\text { Prehospital emergency thoracotomy: is there any indication? Report of five } \\
\text { cases and an algorithm }\end{array}$ & Brasilien & 2003 & 5 \\
\hline Deakin et al. [34] & $\begin{array}{l}\text { From agonal to output: an ECG history of a successful pre-hospital thoraco- } \\
\text { tomy }\end{array}$ & $\begin{array}{l}\text { England } \\
\text { (London) }\end{array}$ & 2007 & 1 \\
\hline Schauer et al. [35] & $\begin{array}{l}\text { The pediatric resuscitative thoracotomy during combat operations in Iraq } \\
\text { and Afghanistan-A retrospective cohort study }\end{array}$ & $\begin{array}{l}\text { Afghanistan/ } \\
\text { Irak }\end{array}$ & 2018 & 6 \\
\hline Schober et al. [36] & $\begin{array}{l}\text { Emergency clamshell thoracotomy in blunt trauma resuscitation: Shelling } \\
\text { the paradigm- } 2 \text { cases and review of the literature }\end{array}$ & Niederlande & 2018 & 2 \\
\hline \multicolumn{5}{|l|}{ ECG Electrocardiogram } \\
\hline
\end{tabular}

Für die Variable Outcome wurde nur in wenigen Publikationen ein definierter Score genutzt. Zur besseren Objektivierbarkeit wurden die Beschreibungen der Outcomes der einzelnen Publikationen von den Autoren dieser Studie an die Glasgow Outcome Scale (GOS) angepasst (• Tab. 2).

Bei mangelnder Datenlage oder widersprüchlichen Informationen in den eingeschlossenen Publikationen wurden der korrespondierende Autor oder die Herausgeber kontaktiert. Hieraus resultierte jedoch kein zusätzlicher Ein- oder Ausschluss von Publikationen.

Die finale Datensammlung wurde von einem zweiten, unabhängigen Autorenteam validiert. Danach wurden mit der Software SPSS studienweise Überlebensraten (in Prozent) zum jeweiligen Outcome berechnet.

\section{Datensynthese und Analyse}

Die deskriptive Auswertung erfolgte mit der Software SPSS (IBM Corp. Released 2016. IBM SPSS Statistics for Windows, Version 24.0. Armonk, NY: IBM Corp.) und umfasst die Darstellung der oben aufgeführten Variablen als relative Häufigkeiten. 


\begin{tabular}{|c|c|c|c|c|c|}
\hline Publications & Outcome Score & Outcome described & $\begin{array}{l}\text { Exact outcome } \\
\text { not reported }\end{array}$ & $\begin{array}{l}\text { No survi- } \\
\text { vors }\end{array}$ & $\begin{array}{l}\text { Glasgow Out- } \\
\text { come Scale (GOS) }\end{array}$ \\
\hline Davies ( $n=71,13$ survivors) & \multirow[t]{4}{*}{-} & - & \multirow[t]{4}{*}{-} & \multirow[t]{4}{*}{-} & - \\
\hline GNO: $n=11$ & & "Good neurologic outcome" & & & 5 \\
\hline BNO: $n=2$ & & \multirow[t]{2}{*}{ „Poor neurologic outcome” } & & & 2 \\
\hline DEAD: $n=58$ & & & & & 1 \\
\hline Corral ( $n=6,1$ survivor) & \multirow[t]{3}{*}{-} & - & \multirow[t]{3}{*}{-} & \multirow[t]{3}{*}{-} & - \\
\hline GNO: $n=1$ & & $\begin{array}{l}\text { "Discharged and returned to } \\
\text { his everyday life" }\end{array}$ & & & 5 \\
\hline DEAD: $n=5$ & & - & & & 1 \\
\hline Matsumoto ( $n=34,0$ survivors) & - & - & - & $\mathrm{x}$ & 1 \\
\hline McKenzie ( $n=1,1$ survivor) & - & $\begin{array}{l}\text { „Released home in good con- } \\
\text { dition" }\end{array}$ & - & - & 5 \\
\hline Puchwein ( $n=1,1$ survivor) & - & $\begin{array}{l}\text { "Er zeigt keine neurologischen } \\
\text { Defizite" }\end{array}$ & - & - & 5 \\
\hline Rudolph ( $n=1,0$ survivors) & - & - & - & $x$ & 1 \\
\hline VanVledder ( $n=33,1$ survivor) & \multirow[t]{3}{*}{-} & - & \multirow[t]{3}{*}{-} & \multirow[t]{3}{*}{-} & - \\
\hline GNO: $n=1$ & & $\begin{array}{l}\text { "Discharged home without } \\
\text { any neurological impairment" }\end{array}$ & & & 5 \\
\hline DEAD: $n=32$ & & - & & & 1 \\
\hline Wall ( $n=1,1$ survivor) & - & $\begin{array}{l}\text { "Discharged home in } 21 \text { days, } \\
\text { neurologically intact" }\end{array}$ & - & - & 5 \\
\hline Hachimi-Idrissi ( $n=33,2$ survivors) & $\begin{array}{l}\text { Ranked in "OPC" } \\
\text { (overall perfor- } \\
\text { mance category) }\end{array}$ & \multirow[t]{4}{*}{-} & \multirow[t]{4}{*}{-} & \multirow[t]{4}{*}{-} & - \\
\hline GNO: $n=1$ & OPC 1 & & & & 5 \\
\hline BNO: $n=1$ & OPC 2 & & & & 4 \\
\hline DEAD: $n=31$ & OPC 5 & & & & 1 \\
\hline Dahmen ( $n=1,1$ survivor) & - & $\begin{array}{l}\text { "Ohne neurologische oder kar- } \\
\text { diologische Einschränkungen" }\end{array}$ & - & - & 5 \\
\hline Belezia ( $n=5,0$ survivor) & - & - & - & $x$ & 1 \\
\hline Deakin ( $n=1$, 1 survivor) & - & $\begin{array}{l}\text { „Made a good recovery and } \\
\text { eventually returned home, } \\
\text { capable of independent living“ }\end{array}$ & - & - & 5 \\
\hline Purkiss ( $n=9,0$ survivors) & - & - & - & $x$ & 1 \\
\hline Schauer ( $n=6,4$ survivors) & - & - & $x$ & - & 0 \\
\hline Schober ( $n=2,0$ survivors) & - & - & - & $x$ & 1 \\
\hline
\end{tabular}

\section{Verzerrungspotenzial}

Da es sich bei den eingeschlossenen Publikationen ausschließlich um Veröffentlichungen mit einem niedrigen Evidenzgrad (Grading Recommendation Score 0) handelt, ist das Verzerrungspotenzial der Publikationen selbst wie auch das der Publikationen untereinander durch die hohe Heterogenität von Populationen, Indikationskriterien und Messmethoden sowie den Mangel von Vergleichsgruppen und großen Fallzahlen als sehr hoch einzuschätzen.
Die Anwendung gängiger Bias-Bewertung-Tools war aufgrund der für ein Review unüblichen Art der eingeschlossenen Publikationen (ausschließlich Fallberichte) nicht in Gänze umsetzbar. Es erfolgten jedoch, soweit auf Fallberichte zutreffend, die Bewertung und Objektivierung mittels eines modifizierten Bias-Bewertungs-Tools (Einsehbar als Onlinmaterial).

\section{Ergebnisse}

\section{Deskriptive Analyse und Review}

Der Prozess der systematischen Literatursuche und -auswahl ist in - Abb. 2, PRISMA-Flussdiagramm, dargestellt. Die internetbasierte Literaturrecherche in 5 Datenbanken ergab 4602 Publikationen (PubMed: 1131, EMABSE: 406, Google Scholar: 1680, SpringerLink: 1383, Cochrane Library: 2). Nach Ausschluss der Publikationen (• Abb. 2) die nicht die Einschlusskriterien erfüllten, re- 


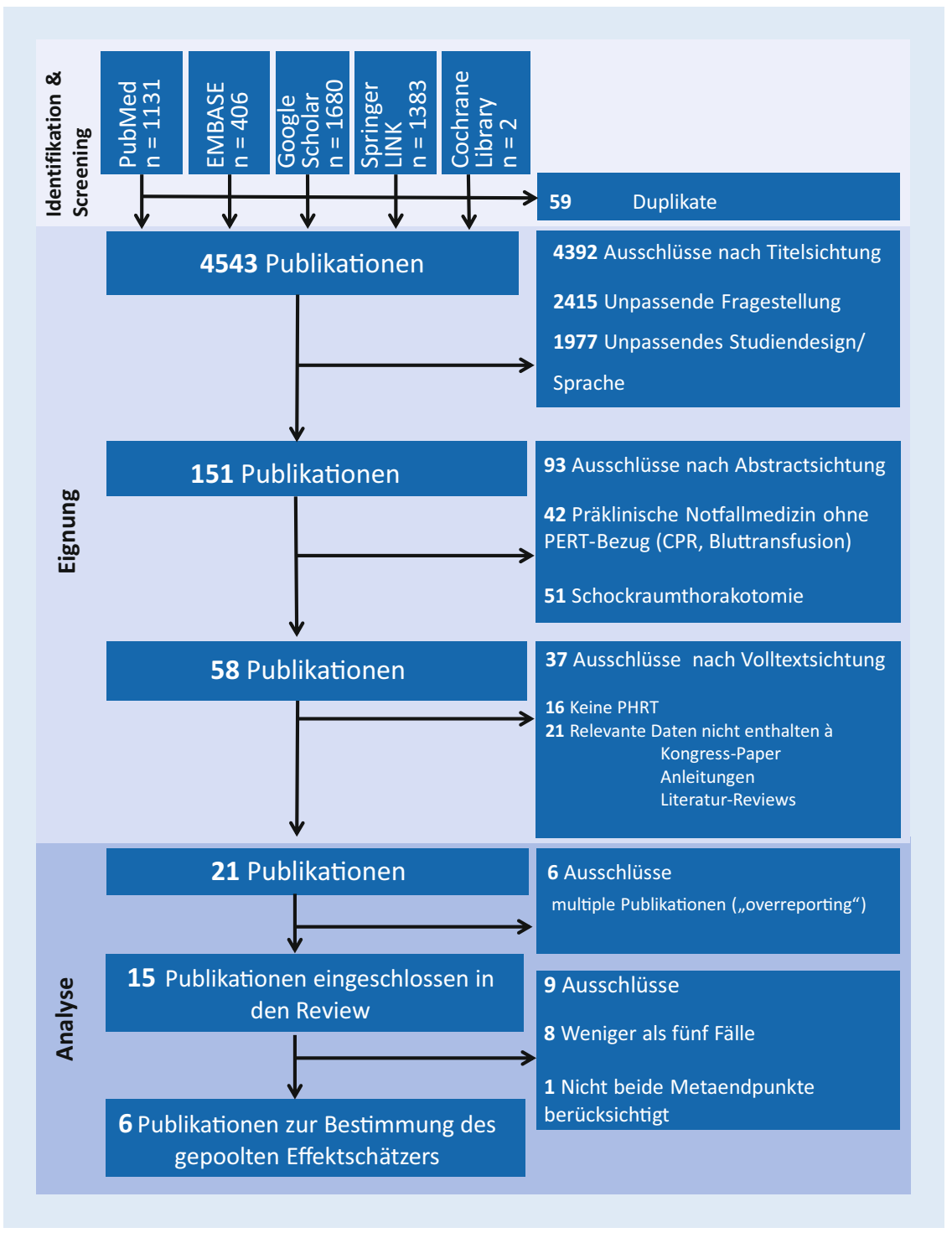

Abb. 2 A Grafische Darstellung des Prozesses für den Ein- und Ausschluss von Studien zur präklinischen Notfallthorakotomie, die zur Erstellung des systematischen Reviews geeignet waren. CPR "Cardiopulmonary Resuscitation"

sultierten 21 Veröffentlichungen mit insgesamt 287 behandelten Patienten, die sich für die weitere Analyse eigneten. Diese wurden weltweit in insgesamt 10 verschiedenen Ländern veröffentlicht, wovon 9 von 21 Publikationen in England publiziert wurden. Deutschland und die Niederlande publizierten je 2 PHRT-Publikationen; die übrigen Berichte stammen aus Österreich, Belgien, Spanien, USA, Brasilien, Japan, Afghanistan, dem Irak und einem ungenannten Einsatzort des US-Militärs (jeweils eine Publikation/Land).

Sechs der 21 Publikationen wurden im Weiteren trotz Vorliegen der einem „double reporting“ und damit einem „publication bias" auszugehen ist. Es wurde in die vorliegende Arbeit deshalb die Publikation mit dem längsten Datenerhebungszeitraum (Davies et al. 1993-2008 [16]) eingeschlossen. $\mathrm{Da}$ lediglich eine weitere Londoner $\mathrm{Pu}$ blikation außerhalb dieses Zeitraums lag, wurden für die deskriptive Analyse nur 2 der 8 Londoner Publikationen berücksichtigt. Somit stützt sich die deskriptive Analyse des vorliegenden Reviews auf insgesamt 15 Publikationen (205 Patienten).

\section{Analyse des Verletzungsmusters}

Das Verletzungsmuster wurde in 11 Publikationen (89 Patienten) für alle eingeschlossenen Fälle berichtet, in 2 Publikationen für einen Teil der Fälle (63 Patienten), und in 2 weiteren Berichten (39 Patienten) konnten den Publikationen zu dieser Variable keinerlei Informationen entnommen werden. Das Verletzungsmuster wurde somit $\mathrm{zu} 50 \%(n=103 / 205)$ der berichteten PHRT-Fälle angegeben.

$57 \%(n=59 / 103)$ der PHRT-Patienten mit berichtetem Verletzungsmuster erlitten penetrierende Verletzungen und $43 \%(n=44 / 103)$ ein stumpfes Trauma.

\section{Analyse der Ursache des Herz-Kreislauf-Stillstands}

14 Publikationen (136 Patienten) boten Informationen zur mutmaßlichen Ursache des TCA. In 12 Publikationen (122 Patienten) wurden Angaben für alle eingeschlossenen Fälle dokumentiert, in 2 Veröffentlichungen für einen Teil der Patienten (14 Fälle), und einer Studie (6 Patienten) waren keine Informationen $\mathrm{zu}$ entnehmen. Bei $66 \%(n=136 / 205)$ der eingeschlossenen Patienten wurden mutmaßliche Ursachen des TCA angegeben.

In $26 \%(n=35 / 136)$ wurde eine Perikardtamponade als Ursache des TCA angegeben, bei ebenso $26 \%(n=35 / 136)$ eine größere thorakale Gefäßverletzung; in $5 \%(7 / 136)$ waren Verletzungen des Lungenparenchyms ursächlich und in $43 \%$ (59/136) andere extrathorakale Verletzungen. 
Analyse des Zeitraums zwischen Herz-Kreislauf-Stillstand und Eintreffen des PHRT-Teams

Eine Dokumentation zum Zeitraum des Herz-Kreislauf-Stillstands bis zum Eintreffen des PHRT-Teams am Patienten erfolgte in 15 Publikationen (76 Patienten). In einer Publikation (ein Patient) wurde eine PHRT wegen Progression der Kreislaufdepression in einer „Periarrest"-Situation bei erhaltenem Restkreislauf durchgeführt.

In $65 \%(50 / 77)$ erlitten die Patienten den TCA im Beisein des anschlieBend thorakotomierenden Notarztes. In $4 \%$ (3/77) erreichte das PHRT-Team den Patienten in $<5 \mathrm{~min}$, in $21 \%(16 / 77)$ in $<10 \mathrm{~min}$, und in $9 \%(7 / 77)$ dauerte es länger als $10 \mathrm{~min}$, bis das Team vor Ort eintraf.

\section{Analyse des Zeitraums zwischen TCA und Beginn der PHRT}

$\mathrm{Zu}$ diesem Zeitraum waren Angaben in 4 Publikationen (4/205 Patienten (2\%)) $\mathrm{zu}$ finden. Diese berichteten alle von einer Zeitspanne zwischen TCA und Beginn der PHRT $<10$ min. 11 Publikationen (201/205 Patienten, $98 \%)$ boten keine Informationen zu diesem Zeitraum.

\section{Analyse des Thorakotomie- verfahrens}

Der gewählte Zugangsweg für die PHRT wurde in 11 Publikationen (88/205 Patienten, $43 \%$ ) angegeben. Dabei erfolgte eine Dokumentation für alle Fälle in 9 Berichten (74 Patienten), Angaben zu einem Teil der eingeschlossenen Fälle in 2 Studien (zu 14 Patienten berichtet, nicht berichtet in 63 Fällen). Vier Publikationen boten keine Information (54 Patienten).

In $53 \%(47 / 88)$ der Fälle wurde eine Clamshell-Thorakotomie als Zugangsweg gewählt und in $47 \%(41 / 88)$ eine linksanterolaterale Thorakotomie durchgeführt.
Analyse des Fachgebiets der
thorakotomierenden Ärzte

Informationen zur Ausbildung bzw. Gebietsbezeichnung der Ärzte, die eine Thorakotomie durchgeführt hatten, konnten 9 Veröffentlichungen (60\%) entnommen werden. Dabei erfolgte eine Angabe der Informationen in 7 Publikationen für alle eingeschlossenen Fälle (80 Patienten); in 6 Publikationen (48 Patienten) fehlten Angaben hierzu. Zwei Studienberichte gaben Informationen für einen Teil der Fälle (für 63 Patienten keine Angaben; für 14 Patienten lagen Informationen vor).

In $71 \%$ der Fälle $(n=66 / 94)$ wurden die Thorakotomien von Notärzten ohne chirurgische Gebietsbezeichnung durchgeführt. In $27 \%(n=25 / 94)$ wurden die Thorakotomien von Anästhesisten, in $29 \%(n=28 / 94)$ von Chirurgen und in $44 \% \quad(n=41 / 94)$ von ,emergency physicians “ mit eigenständiger Gebietsbezeichnung durchgeführt (in der überwiegenden Zahl der beteiligten Länder ist die (prä-)klinische Notfallmedizin als eigenständige, primäre Gebietsbezeichnung anerkannt [37]).

\section{Analyse des Outcome der Patienten nach PHRT}

Das Outcome der Patienten nach TCA und erfolgter PHRT wurde hinsichtlich verschiedener Zeitpunkte untersucht, welche in $-\mathrm{Abb}$. 3 dargestellt sind. Allen Berichten konnten Informationen zum Überleben der Patienten nach TCA und PHRT entnommen werden. In 13 Publikationen (53/205 Patienten (26\%)) wurde das Erreichen eines „return of spontaneous circulation“ (ROSC) beschrieben. Ebenso enthielten 13 Publikationen (42/205 Patienten, 21\%) Informationen zu Patienten, welche lebend das Krankenhaus erreichten. Informationen zur Entlassung der Patienten aus dem Krankenhaus konnten 10 Publikationen (26 Patienten, $13 \%$ bzw. $49 \%$ aller PHRT-Patienten, die jemals einen ROSC erreichten) entnommen werden. Dabei wiesen 9 Publikationen Angaben zur Entlassung der Patienten mit gutem neurologischen Outcome (GOS: 5) auf (19/26 Patienten, $73 \%$ der Überleben- den), 2 machten Angaben zur Entlassung mit schlechtem neurologischen Outcome (GOS: 2-4) (3/26 Patienten, 12\% der Überlebenden), und einer Publikation (4/26 Patienten, $15 \%$ der Überlebenden) konnte keine Angabe zum neurologischen Outcome (GOS: 0) entnommen werden. Der Anteil der Patienten mit gutem neurologischen Outcome aller eingeschlossenen PHRT-Patienten betrug $9 \%(n=19 / 205)$.

Zusammenfassend konnte in der vorliegenden deskriptiven Auswertung aller eingeschlossenen Patienten gezeigt werden, dass 26\% ( $n=53 / 205$ Patienten) ein ROSC nach PHRT erreichten. 79\% $(n=42 / 53)$ dieser Patienten mit ROSC nach PHRT erreichten auch lebend das Krankenhaus. Mehr als jeder zweite dieser Patienten $(n=26 / 53)$ konnte später auch lebend das Krankenhaus verlassen, davon $73 \%(n=19 / 26)$ in gutem neurologischen Outcome.

Randomisierte klinische Studien mit Kontrollgruppen oder gar Randomisierung konnten zur PHRT in keiner Publikation gefunden werden. Eine der 21 eingeschlossenen Publikationen zeigte eine Vergleichsgruppe, in der die Ergebnisse der Patienten nach TCA und PHRT mit Patienten nach TCA und StandardACLS-Therapie verglichen wurden [35]. Schauer et al. konnten in dieser Untersuchung zeigen, dass 13 PHRT-Patienten ein signifikant häufigeres Überleben nach TCA hatten als 66 Patienten mit gleichem Verletzungsmuster („Matchedpair“-Analyse), die mittels StandardACLS-Therapie behandelt worden waren ( $31 \%$ vs. $9 \%)$.

Ein Matched-Pair-Vergleich zwischen PHRT und Emergency Department Thoracotomy (EDT) Patienten wurde in 3/21 Publikationen publiziert [22, 25, 35]. Matsumoto et al. und Purkiss et al. konnten dabei in keiner der Gruppen Überlebende bis zur Krankenhausentlassung feststellen. Schauer et al. berichten in einer kleinen Fallserie, dass in der PHRT Gruppe 4/6 Patienten und in der EDT-Gruppe 0/7 überlebten.

\section{Diskussion}

Das vorliegende Review gibt einen umfassenden systematischen Überblicküber 


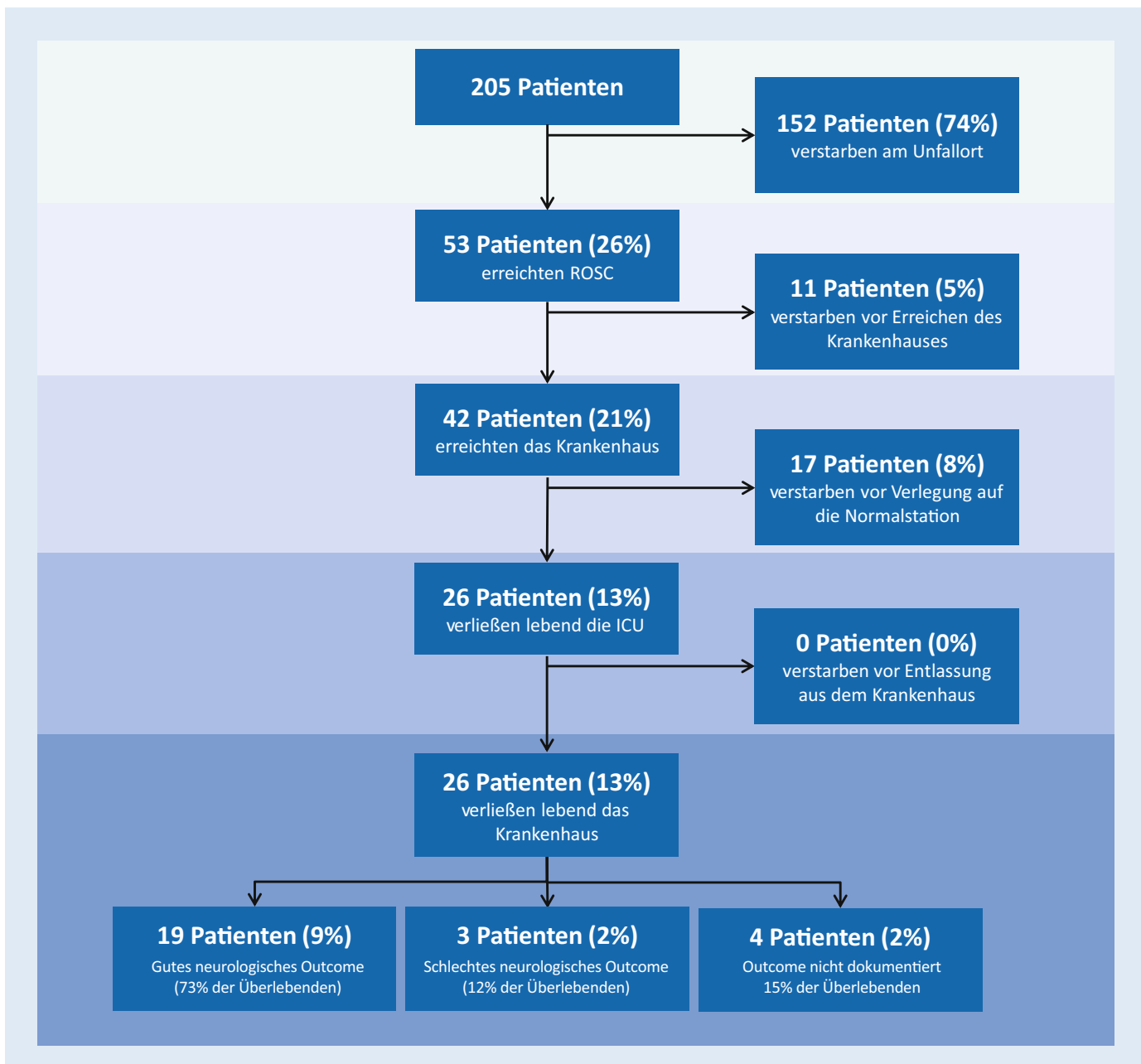

Abb. $3<$ Flowchart zum Outcome nach PHRT - Darstellung der Anzahl von Patienten $(N)$ mit erzieltem Outcome, im zeitlichen Verlauf der Behandlung. Darstellung für 15 Studienberichte (205 Patienten) im systematischen Review. ROSC "Return Of Spontaneus Circulation"

die vollständige englisch- und deutschsprachige PHRT-Literatur. Die erste gefundene Publikation stammt aus dem Jahr 1988, die jüngste aus dem Jahr 2018. Für 205 Patienten (aus 15 Publikationen), bei denen präklinisch eine PHRT bei TCA durchgeführt wurde, untersucht das vorliegende Review dabei Indikation und Durchführung der Maßnahmen sowie das Outcome der Patienten.

Die präklinische Versorgung von schwer verletzten Patienten stellt weltweit und auch in Deutschland nach wie vor eine große Herausforderung dar. Die hohe Sterberate in der Präklinik von bis zu $60 \%$ aller verstorbenen Traumapatienten in Deutschland wirft die Frage nach Vermeidbarkeiten und $\mathrm{zu}$ ändernden Behandlungsstrategien auf [10, 38].

Buschmann und Kleber et al. konnten inzwischen in mehreren Arbeiten zeigen, dass sich in der Traumaversorgung die größte Anzahl potenziell und definitiv vermeidbarer Behandlungsfehler in der prähospitalen Notfallmedizin ereignet $[10,38]$. Im Vordergrund der Fehleranalysen $\mathrm{zu}$ potenziell und definitiv vermeidbaren Traumatodesfällen in der Präklinik steht das hohe lebensrettende Potenzial der präklinischen Durchführung basaler Maßnahmen, wie beispielsweise die suffiziente externe Blutungskontrolle, etwa durch die Anwendung von effektiven Druckverbänden, Tourniquet, Gefäßklemmen oder Einlage eines Foley-Katheters in blutende, nichtkomprimierbare Stichkanäle.

Neben einem generellen Mangel an basalen Traumamanagementkompetenzen (fehlende klinische Ganzkörperuntersuchung, grobe Missachtung der ABCDE-Regel) zeigten sich in diesen Auswertungen auch andere schwerwiegende Behandlungsmängel wie z.B. eine feh- lende bzw. insuffiziente Dekompression eines Spannungspneumothorax.

Inzwischen wurde seit diesen Berichten - auch bedingt durch eine erhöhte Aufmerksamkeit nach mehreren Terrorund Amoklagen in Europa - eine Reihe von Maßnahmen zur Verbesserung der Versorgungsqualität in Deutschland ergriffen. Beispielsweise sei hier die Implementierung des "trauma care bundle“ der Deutschen Gesellschaft für Unfallchirurgie (DGU) in etlichen regionalen "standard operating procedures" (SOP) für den Rettungsdienst [39] genannt.

Auch die Einführung des Berufsbildes „Notfallsanitäter“ hat bereits heute erkennbar zu einer weiteren Professionalisierung und Standardisierung der präklinischen Notfallmedizin beigetragen. Es ist jedoch davon auszugehen, dass jenseits der inzwischen ergriffenen Maßnahmen weitere Anstrengungen, insbesondere in der Schulung des not- 
ärztlichen Personals in Deutschland, unternommen werden müssen, um insbesondere Traumapatienten mit erhaltenem Kreislauf durch einfache und standardisierte Versorgungsverfahren vor einem Kreislaufstillstand zu bewahren.

Neben den genannten Mängeln bei der Versorgung von Traumapatienten mit Kreislauf stellt sich zusätzlich die Frage, welcher Maßnahme(n) es bedarf, um hinsichtlich der Reanimation nach Trauma die Empfehlungen der ERC-Leitlinie (einschließlich maximalinvasiver Maßnahmen wie eine PHRT) in der präklinischen Notfallmedizin in Deutschland umzusetzen.

Kleber et al. konnten in ihren Untersuchungen des überwiegenden Teils aller Traumaverstorbenen eines Jahres (2010 für Berlin) herausarbeiten, dass mindestens 4 der 26 potenziell vermeidbaren Todesfälle möglicherweise durch eine PHRT hätten gerettet werden können. Rechnet man diese Zahl auf die Gesamtbevölkerung in Deutschland hoch, entspräche dies bei allen statistischen Ungenauigkeiten und regionalen Unterschieden mehr als 90 potenziell durch eine PHRT geretteten Menschenleben/ Jahr. Unklar bleibt bei der Betrachtung von Kleber et al., wie groß der Anteil der Patienten ist, die jenseits einer thorakalen Verletzung $(n=4)$ durch andere, potenziell reversible Ursachen (ausgedehnte oder massive intraabdominelle bzw. intrapelvine Blutungen) potenziell durch eine PHRT gerettet werden könnten (beispielsweise durch direkte Blutungskontrolle, Abklemmen der Aorta und direkte Herzmassage; - Infobox 1).

Vor dem Hintergrund der Fehlerquote in der präklinischen Traumaversorgung und der Inzidenz reversibler Ursachen eines traumatisch bedingten HerzKreislauf-Stillstands sind die Autoren der Auffassung, dass forcierte Maßnahmen zur verbesserten allgemeinen rettungsdienstlichen Kompetenz in der Traumaversorgung unerlässlich und von hoher medizinischer, aber auch gesamtgesellschaftlicher Dringlichkeit sind.

Darüber hinaus scheint es sinnvoll, insbesondere in Gebieten mit hoher Besiedlungsdichte (zumindest auf Spezialrettungsmitteln wie beispielsweise der
Luftrettung), das Personal zur Durchführung einer PHRT gemäß der ERCLeitlinien (• Abb. 1) zu schulen.

In der ERC-Leitlinie wird die durchschnittliche Überlebenswahrscheinlichkeit aller Patienten nach TCA und PHRT mit $15 \%$ angegeben. Dies deckt sich mit den Ergebnissen des vorliegenden Reviews (13\% Überleben nach PHRT bis zur Krankenhausentlassung in der deskriptiven Analyse). Diese Ergebnisse stehen im deutlichen Kontrast zu den schlechten Überlebensraten (3-4\% bis zur Krankenhausentlassung) im Fall von kardiopulmonaler Reanimation beim TCA ohne konsekutive Thoraxdekompression und Notfallthorakotomie [40].

Bisher werden die von der ERC- und S3-Leitlinie [6, 41] für die Traumareanimation empfohlenen Maßnahmen in Deutschland nur unzureichend [9] und die präklinische Notfallthorakotomie im deutschsprachigen Raum bis auf wenige Ausnahmen [26, 31, 42] kaum durchgeführt. Das häufige Unterlassen empfohlener Maßnahmen der ERCLeitlinie für die Traumareanimation ist möglicherweise für das schlechte Outcome der Traumareanimationspatienten in Deutschland mitverantwortlich. So zeigten Jakisch et al. bei einer Analyse aller im Zeitraum 2000-2017 ins Deutsche Reanimationsregister eingeschlossenen Patienten nach traumatischer Reanimation, dass $10 \%$ die ersten $24 \mathrm{~h}$ nach Krankenhausaufnahme überlebten und nur $4 \%$ das Krankenhaus lebend verließen. Die Notwendigkeit, hier zu einem konzeptionellen Umdenken beim TCA in Deutschland zu kommen, unterstreichen Leis et al., in dem sie zeigen konnten, dass der TCA, im Vergleich zum kardial-bedingten Herz-KreislaufStillstand, mit einem besseren neurologischen Outcome assoziiert ist [43].

In den Leitlinien und in vielen der eingeschlossenen Publikationen zeigten sich hier immer wieder weit auseinandergehende Werte für das Outcome nach stumpfem und penetrierendem Trauma $(35 \%$ nach penetrierendem Trauma, 0-2\% nach stumpfem Trauma $[6,22,44])$. Diese Dissoziation konnte innerhalb des vorliegenden Reviews nicht geprüft werden, da viele der Publikationen mit gemischt penetrierend- stumpfen Traumapatienten bei Angabe des Outcome nicht zwischen der vorangegangenen Verletzungsart der Überlebenden differenzierten. Jedoch gab es einen deutlichen Trend hin zu einer niedrigeren Überlebenswahrscheinlichkeit in Publikationen nach stumpfem Thoraxtrauma [22]. Ungeachtet dessen kann davon ausgegangen werden, dass insbesondere Patienten mit penetrierendem Thoraxtrauma unter gegebenen Indikationskriterien von einer PHRT profitieren können [6].

Innerklinisch bzw. im Schockraum ist die Notfallthorakotomie weltweit deutlich etablierter: Das aktuelle und umfassendste Review von Nevins et al. zeigt für 3251 Patienten nach EDT im Schockraum eine Überlebensrate von $8,5 \%$ ( $n=277)$ [45]. Dies steht im Kontrast zu den Daten des vorliegenden Reviews, in dem die Überlebensrate mit $13 \%$ trotz eingeschränkterer Behandlungsbedingungen in der Präklinik (Verfügbarkeit von Equipment, Expertise und gegebenem Environment) wesentlich höher liegt.

Zwingmann et al. berichteten von Ergebnissen aus dem Bereich der Reanimationsforschung in Deutschland. Diese zeigen, dass im Falle eines TCA bei effektiven und erfolgreichen Reanimationsanstrengungen in der Präklinik, diese Patienten die besten Überlebenschancen haben, gefolgt von Patienten, welche im Schockraum reanimationspflichtig wurden. Patienten, bei denen die Reanimationsbemühungen aus der Präklinik unter laufender CPR in den Schockraum verlagert wurden, hatten die schlechteste Überlebenswahrscheinlichkeit und das schlechteste neurologische Outcome [5].

Auch ATLS $^{\circledR}$ als weltweit verbreitetes Kurskonzept der klinischen Traumaversorgung hat mit der neuesten 10. Edition die EDT im Schockraum in einem eigenen Traumareanimationsalgorithmus in das Kurskonzept aufgenommen [46], ebenso wie der European Trauma Course $\left(\right.$ ERC $\left.^{\circledR}\right)$ in seiner 4. Version [47]. In der Vergangenheit sind präklinische Traumakursformate wie etwa PHTLS ${ }^{\circledR}$ oder ITLS ${ }^{\circledR}$ häufig den Entwicklungen von ATLS $^{\circledR}$ gefolgt, sodass abzuwarten bleibt, ob hier insbesondere hinsichtlich notarztgestützter präklinischer Trauma- 
versorgungskonzepte auch die PHRT in die Kurskonzepte der bekanntesten präklinischen Traumakursformate Einzug hält.

Trotz bisher fehlender flächendeckender Implementierung haben sich inzwischen in Deutschland insbesondere im Bereich der Luftrettung [26, 31] mehrere Standorte auf den Weg gemacht, entsprechende Versorgungskonzepte $\mathrm{zu}$ etablieren. Auch eine wachsende Zahl an Kursangeboten sorgt für eine intensivere Auseinandersetzung mit dem Thema PHRT in der Notfallrettung [48-50]. Puchwein et al. konnten hinsichtlich derartiger Kurskonzepte zu TCA bzw. PHRT zeigen, dass nach einem Kadaverkurs und Tutorial der Eingriff auch von Notärzten mit nichtchirurgischer Gebietsbezeichnung in $<3 \mathrm{~min}$ ohne statistisch signifikanten Qualitätsunterschied sicher durchgeführt werden kann [13]. Dies deckt sich mit den Daten des vorliegenden Reviews, das zeigten konnte, dass die überwiegende Zahl an publizierten PHRT-Fällen nicht von Notärzten mit chirurgischer Gebietsbezeichnung durchgeführt wurde. Jenseits der verfügbaren Kursangebote ist inzwischen international auch eine ganze Serie an praktischen Anleitungen und SOP für die Durchführung von PHRT für die präklinische Notfallmedizin publiziert, welche deutlich machen, wie eine pragmatische Implementierung der PHRT in die TCA-Reanimation-Algorithmen erfolgen kann $[11,18,51]$.

\section{Aktuell vorhandene \\ PHRT-Übersichtsliteratur}

Trotz vielfacher Erwähnung der präklinischen Notfallthorakotomie in Leitlinien und Manuals $[6,51,52]$ fehlt es bisher an hochwertigen, prospektiven, randomisierten Studien unter Einschluss der erforderlichen Patientenzahl. Anhaltende ethische Restriktionen und fehlende ausreichende Fallzahlen haben bisher randomisierte kontrollierte Studien unmöglich gemacht und werden auch in Zukunft nicht durchführbar sein. Somit kommt den vorhandenen eingeschlossenen Einzelfallberichten und retrospektiven Fallserien trotz aller umfangreichen Limitationen eine große Bedeutung zu.
Eine systematische Übersicht über die (in Englisch oder Deutsch) vorhandenen Publikationen zur präklinischen Notfallthorakotomie gab es bislang nur in Auszügen. Hafstad et al. [53] publizierten 2017 einen Überblick über 6 PHRT-Studien, jedoch ohne vergleichende Analyse der PHRT-Fälle bezüglich der Art des Traumas, Prozedere oder Outcome. Auch Rabinovici et al. [54] fassten 2013 einen Teil der PHRT-Literatur zusammen, ebenso ohne die Analyse der gemeinsamen Faktoren. Das aktuelle Review von Nevins et al. [55] aus dem Jahr 2018 umfasst 12 PHRT-Publikationen mit einem Schwerpunkt auf den Publikationen der London HEMS. Neun weitere, vorhandene Publikationen, die in der vorliegenden Arbeit untersucht wurden, sowie das neurologische Outcome wurden durch Nevins nicht berücksichtigt bzw. verwendet.

\section{Limitationen dieses Reviews}

Bis heute steht nur eine sehr limitierte Anzahl von Veröffentlichungen für die Fragestellung bezüglich des Outcome nach präklinischer Thorakotomie zur Verfügung bzw. weisen diese - nach Studiendesign - einen geringen Evidenzgrad auf (ausschließlich retrospektive Studien und Fallberichte).

Bei einer insgesamt schwachen Publikations- und Datenlage gehen die Autoren von einem nichtunerheblichen $P u b$ lication bias aus. So ist beispielsweise ein „underreporting“ dort zu vermuten, wo einerseits eine PHRT bisher nur vereinzelt oder nicht erfolgreich durchgeführt wurde, aber auch andererseits dort, wo - wie in London durch die London's Air Ambulance (>2/Woche laut mündlicher Anfrage der Autoren) oder den Queensland Ambulance Service - die PHRT bereits seit vielen Jahren etabliert ist und seit 2008 keinerlei neuere Daten über die inzwischen häufige und standardisierte Durchführung von PHRT publiziert wurden.

Eine wesentliche Einschränkung ergab sich ebenfalls im Hinblick auf die Analyse der Zusammenhänge zwischen den untersuchten Faktoren und dem Outcome innerhalb dieses Reviews. So ließ sich beispielsweise für eingeschlos- sene Publikationen mit gemischt penetrierenden und stumpfen Traumata in den meisten Fällen nicht nachvollziehen, welche der Patienten überlebten. Somit ließ sich für die meisten untersuchten Faktoren lediglich ein nummerisches Vorkommen, jedoch kein einzelfaktorbezogener Einfluss auf das Outcome nachweisen.

Trotz aller fortbestehender Grenzen bei der Erarbeitung hochwertiger Evidenz im Kontext von TCA und PHRT sollten insbesondere im Bereich der Registerforschung Anstrengungen unternommen werden, um zukünftig ein fundierteres Verständnis für Maßnahmen zur Verbesserung der präklinischen Traumaversorgung, einschließlich Reanimation und PHRT, zu erlangen.

\section{Resümee}

Vor dem Hintergrund intensiver Diskussionen entlang der ERC-Reanimationsleitlinie über den Wert der PHRT bei der Versorgung des TCA ist das vorliegende Review die unserer Kenntnis nach bislang umfassendste systematische Übersichtsarbeit zu PHRT im deutsch- und englischsprachigem Raum.

Für das Überleben und Outcome ist die Durchführung aller von der ERC-Leitlinie empfohlenen Maßnahmen zur Therapie der reversiblen Ursachen des traumatischen Kreislaufstillstands - auch in der Präklinik - von entscheidender Bedeutung. Eine präklinische Notfallthorakotomie ist als Teil dieser Maßnahmen mit einer 12 \%igenÜberlebenswahrscheinlichkeit und einer hohen Rate von gutem neurologischen Outcome vergesellschaftet. Dennoch ist diese Maßnahme in der Präklinik im deutschsprachigen Raum, anders als in einer Reihe europäischer Nachbarländer, bisher kaum etabliert. Umfangreiche, gesamtgesellschaftliche Anstrengungen sind erforderlich, um die PHRT mittels umfangreicher Aufklärung und regelmäßiger Schulung im deutschsprachigen Raum zu etablieren und die Anzahl vermeidbarer Traumatodesfälle zu reduzieren. Dabei kommt der präklinischen Notfallmedizin und insbesondere der Reduktion vermeidbarer Behandlungsfehler eine große Bedeutung zu. Die 
vorliegende Arbeit kann dabei helfen, aufbauend auf diesen Maßnahmen. die PHRT im deutschsprachigen Raum entlang den Leitlinienempfehlungen systematisch, beispielsweise auf speziellen Rettungsmitteln wie der Luftrettung, in Ballungszentren zu etablieren.

\section{Korrespondenzadresse}

\section{J. Dahmen}

Ärztliche Leitung Rettungsdienst Berlin, Fakultät für Gesundheit, Department Humanmedizin, Universität Witten/Herdecke Alfred-Herrhausen-Straße 50, 58455 Witten, Deutschland janosch.dahmen@berliner-feuerwehr.de

Funding. Open Access funding provided by Projekt DEAL.

\section{Einhaltung ethischer Richtlinien}

Interessenkonflikt. B. A. Leidel, T. Malysch und J. Dahmen erhielten Reisekostenerstattungen und Honorare für Instruktorentätigkeiten von Notfallmedizinkurs GbR, die als Drittmittel ausschließlich für wissenschaftliche Projekte verwendet werden. J. Schimrigk, C. Baulig, C. Buschmann, J. Ehlers, C. Kleber, S. Knippschild und E. Steinhausen geben an, dass kein Interessenkonflikt besteht.

\section{Für diesen Beitrag wurden von den Autoren keine} Studien an Menschen oder Tieren durchgeführt. Für die aufgeführten Studien gelten die jeweils dort angegebenen ethischen Richtlinien.

Open Access. Dieser Artikel wird unter der Creative Commons Namensnennung 4.0 International Lizenz veröffentlicht, welche die Nutzung, Vervielfältigung, Bearbeitung, Verbreitung und Wiedergabe in jeglichem Medium und Format erlaubt, sofern Sie den/die ursprünglichen Autor(en) und die Quelle ordnungsgemäß nennen, einen Link zur Creative Commons Lizenz beifügen und angeben, ob Änderungen vorgenommen wurden.

Die in diesem Artikel enthaltenen Bilder und sonstiges Drittmaterial unterliegen ebenfalls der genannten Creative Commons Lizenz, sofern sich aus der Abbildungslegende nichts anderes ergibt. Sofern das betreffende Material nicht unter der genannten Creative Commons Lizenz steht und die betreffende Handlung nicht nach gesetzlichen Vorschriften erlaubt ist, ist für die oben aufgeführten Weiterverwendungen des Materials die Einwilligung des jeweiligen Rechteinhabers einzuholen.

Weitere Details zur Lizenz entnehmen Sie bitte der Lizenzinformation auf http://creativecommons.org/ licenses/by/4.0/deed.de.

\section{Literatur}

1. Deutsche Gesellschaft für Unfallchirurgie (2018) Jahresbericht 2018 - TraumaRegister DGU ${ }^{\circledR}$ für den Zeitraum 2017

2. Navarro $S$ et al (2014) Impact of ATLS training on preventable and potentially preventable deaths. World JSurg 38(9):2273-2278

3. Melniker LA et al (2006) Randomized controlled clinical trial of point-of-care, limited ultrasonography for trauma in the emergency department: the first sonography outcomes assessment program trial. Ann Emerg Med 48(3):227-235

4. Kleber C et al (2014) Requirement for a structured algorithm in cardiac arrest following major trauma: epidemiology, management errors, and preventability of traumatic deaths in Berlin. Resuscitation 85(3):405-410

5. Zwingmann J et al (2016) Outcome and predictors for successful resuscitation in the emergency room of adult patients in traumatic cardiorespiratory arrest. Crit Care 20(1):282

6. Truhlar A et al (2015) European resuscitation council guidelines for resuscitation 2015: section 4. cardiac arrest in special circumstances. Resuscitation 95:148-201

7. Truhláŕ A, Deakin C, Soar J et al (2015) Kreislaufstillstand in besonderen Situationen. Kapitel 4 der Leitlinien zur Reanimation 2015 des European Resuscitation Council. Notfall Rettungsmed 18:833-903

8. Truhláŕ A, Deakin CD, Soar J et al (2017) Erratum zu: Kreislaufstillstand in besonderen Situationen. Kapitel 4 der Leitlinien zur Reanimation 2015 des European Resuscitation Council. Notfall Rettungsmed 20:540-542

9. Ondruschka B et al (2017) Additional emergency medical measures in trauma-associated cardiac arrest. Anaesthesist 66(12):924-935

10. Kleber $C$ et al (2013) Trauma-related preventable deaths in Berlin 2010: need to change prehospita management strategies and trauma management education. World J Surg 37(5):1154-1161

11. Clinical Quality \& Patient Safety Unit, Queensland Ambulance Service, Queensland Government (2018) Clinical practice procedures: trauma/thoracotomy. https://ambulance.qld.gov. au/clinical.html.Zugegriffen:28. Apr. 2018

12. Rehn M et al (2018) Resuscitative thoracotomy: a practical approach. Surgery 36(8):424-428

13. Puchwein P et al (2015) Clamshell thoracotomy and open heart massage - a potential life-saving procedure can be taught to emergency physicians: an educational cadaveric pilot study. Injury 46(9):1738-1742

14. The Cochrane Collaboration (2011) Cochrane handbook for systematic reviews of interventions. http://handbook-5-1.cochrane.org/. Zugegriffen: 25. Febr. 2019

15. Liberati A et al (2009) The PRISMA statement for reporting systematic reviews and meta-analyses of studies that evaluate healthcare interventions: explanation and elaboration. BMJ 339:b2700

16. Davies GE, Lockey DJ (2011) Thirteen survivors of prehospital thoracotomy for penetrating trauma: a prehospital physician-performed resuscitation procedure that can yield good results. J Trauma 70(5):E75-8

17. Athanasiou Tet al (2004) Emergency thoracotomy in the pre-hospital setting: a procedure requiring clarification. Eur JCardiothoracSurg 26(2):377-386

18. CoatsTJ, KeoghS, ClarkH,NealM(2001)Prehospital resuscitative thoracotomy for cardiac arrest after penetrating trauma: rationale and case series. JTrauma 50(4):670-673

19. Corral E et al (2007) A successful emergency thoracotomy performed in the field. Resuscitation 75(3):530-533

20. Craig R et al (1999) On scene thoracotomy: a case report. Resuscitation 40(1):45-47

21. Keogh SP et al (1996) Survival following prehospital arrest with on-scene thoracotomy for a stabbed heart. Injury 27(7):525-527

22. Matsumoto $\mathrm{H}$ et al (2009) Role of resuscitative emergency field thoracotomy in the Japanese helicopter emergency medical service system. Resuscitation 80(11):1270-1274

23. McKenzie MR et al (2016) A case of prehospital traumatic arrest in a US special operations soldier. Care from point of injury to full recovery. J Spec Oper Med 16(3):93-96

24. Puchwein P et al (2013) Erfolgreiche präklinische Thorakotomie bei einem 17-jährigen Mann. Unfallchirurg. https://doi.org/10.1007/s00113013-2484-0

25. Purkiss SF et al (1994) Efficacy of urgent thoracotomy for trauma in patients attended by a helicopter emergency medical service. J R Coll Surg Edinb 39(5):289-291

26. Rudolph M, Schneider NRE, Popp E (2017) Clamshell-Thorakotomie nach thorakalen Messerstichen. Unfallchirurg 120(4):344-349

27. Van Vledder MG et al (2017) Out of hospital thoracotomy for cardiac arrest after penetrating thoracic trauma. Injury 48(9):1865-1869. https:// doi.org/10.1016/j.injury.2017.04.002

28. Wall MJ et al (1994) Successful roadside resuscitative thoracotomy: case report and literature review. JTrauma 36(1):131-134

29. Wright KD et al (2002) Cardiac tamponade: a case of kitchen floor thoracotomy. Emerg Med J 19(6):587-588

30. Hachimi-Idrissi S et al (1997) Open chest cardiopulmonary resuscitation in out-of-hospital cardiac arrest. Resuscitation 35(2):151-156

31. Dahmen J et al (2018) Erfolgreiche präklinische Notfallthorakotomie nach stumpfem Thoraxtrauma - Fallbericht mit Darstellung gewonnener Erkenntnisse. Unfallchirurg 121(10):839-849

32. Lockey D et al (2006) Traumatic cardiac arrest: who are the survivors? Ann Emerg Med 48(3):240-244

33. Belezia BF et al (2003) Prehospital emergency thoracotomy: is there any indication? Report of five cases and an algorithm. Crit Care 7(3):P114

34. Deakin CD et al (2007) From agonal to output an ECG history of a successful pre-hospital thoracotomy. Resuscitation 75(3):525-529

35. Schauer SG et al (2018) The pediatric resuscitative thoracotomy during combat operations in Iraq and Afghanistan-a retrospective cohort study. Injury 49(5):911-915

36. Schober P et al (2018) Emergency clamshell thoracotomy in blunt trauma resuscitation: shelling the paradigm-2 cases and review of the literature.Clin Case Rep 6(8):1521-1524

37. Dißmann PD (2015) Preparing for the European board examination in emergency medicine

38. Buschmann C et al (2013) Vermeidbare Todesfälle nach Trauma. Notarzt p:91-98

39. Matthes $\mathrm{G}$ et al (2015) Wesentliche Maßnahmen zur prähospitalen Versorgung schwerverletzter Patienten. Unfallchirurg 118(8):652-656

40. Zwingmann J et al (2012) Survival and neurologic outcome after traumatic out-of-hospital cardiopulmonary arrest in a pediatric and adult population: a systematic review. Crit Care 16(4):R117 
41. Lendemans S, Ruchholtz S (2012) S3-Leitlinie Polytrauma/Schwerverletzten-Behandlung. Unfallchirurg 115(1):14-21

42. Puchwein P et al (2014) Successful preclinical thoracotomy in a 17-year-old man. Unfallchirurg 117(9):849-852

43. Leis CC et al (2013) Traumatic cardiac arrest: should advanced life support be initiated? J Trauma Acute Care Surg 74(2):634-638

44. Burlew CC et al (2012) Western trauma association critical decisions in trauma: resuscitative thoracotomy. JTrauma Acute Care Surg 73(6):1359-1363

45. Nevins EJ et al (2019) A systematic review of 3251 emergency department thoracotomies: is it time for a national database? Eur J Trauma Emerg Surg 45(2):231-243

46. Sharon Henry M (2018) ATLS 10th edition offers new insights into managing trauma patients. http://bulletin.facs.org/2018/06/atls-10thedition-offers-new-insights-into-managingtrauma-patients/.Zugegriffen:29. April 2018

47. European Resuscitation Council (2019) Europoean trauma course. https://www.erc.edu/courses/ european-trauma-course. Zugegriffen: 19. Okt. 2019

48. Schneider N et al (2018) INTECH Advanced: REBOA, Perikardiozentese und Clamshell-Thorakotomie. Notf Rett Med. https://doi.org/10.1007/s10049018-0475-y

49. Deutsche Gesellschaft für Unfallchirurgie (2019) DSTC $^{\text {TM: }}$ definitive surgical trauma care

50. Leidel BA et al (2019) „Prehospital and Emergency Department Resuscitative Thoracotomy $\left(\right.$ PERT) ${ }^{\prime \prime}$ - Kurs nach Vorbild des Kurses des britischen Royal College of Surgeons. https://www.notfallmedizinkurs.de/pertnotfallthorakotomie/.Zugegriffen:29. April 2018
51. Wise D et al (2005) Emergency thoracotomy: "how to do it". Emerg Med J 22(1):22-24

52. Chalkias A (2009) Prehospital emergency thoracotomy: when to do it? AJP Rep 7(4):990356

53. Hafstad E, Tjelle TE, Sæterdal I (2017) Prehospital emergency thoracotomy in traumatic cardiac arrest: systematic literature search with sorting. Norwegian Institute of Public Health, Oslo, S3. ISBN 978-82-8082-848-4

54. Rabinovici R, Bugaev N (2014) Resuscitative thoracotomy: an update. Scand J Surg 103(2):112-119

55. Nevins EJ et al (2018) Should pre-hospital resuscitative thoracotomy be reserved only for penetrating chest trauma? Eur J Trauma Emerg Surg 44(6):811-818

\section{Aktuelle Operationstechniken im Video}

Reichen Sie Ihr Video für Der Unfallchirurg jetzt ein!

Im Mittelpunkt des Beitrags steht Ihr Video zu aktuellen Operationstechniken. Es werden bewährte operative Techniken dargestellt. Neben der Vermittlung aller wichtigen prä-, intraund postoperativen Details sowie den Hinweisen auf mögliche Fehler und Gefahren liegt der Schwerpunkt auf einer genauen Darstellung der jeweiligen Operationsschritte im Video.

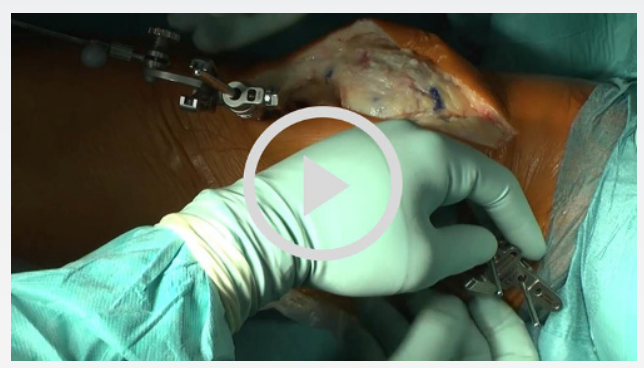

Nutzen Sie das Medium des Videos um komplizierte Vorgehensweisen leicht verständlich zu präsentieren. Bitte reichen Sie zu Ihrem Video einen kurzen begleitenden Beitrag ein, der die wesentlichen Punkte des Videos zusammenfasst.

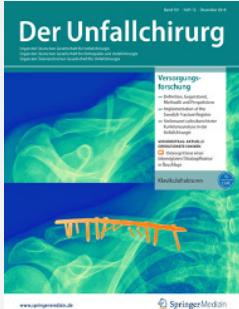

Weitere Informationen zur Einreichung sowie einen detaillierten Leitfaden erhalten Sie auf www.DerUnfallchirurg.de.

Reichen Sie Ihren Videobeitrag online ein unter www.editorialmanager.com/deun/ 